\title{
Spectral Behavior of Quasi Periodic Potentials*
}

\author{
P. Sarnak \\ Courant Institute of Mathematical Sciences, 251 Mercer Street, New York, NY 10012, USA
}

\begin{abstract}
Spectral properties of Schrödinger operators of the type $H_{\varepsilon}=$ $-\Delta+\varepsilon V$, where $\Delta$ is the Laplacian, $V$ a quasiperiodic potential and $\varepsilon$ a coupling constant, are developed. $V$ is taken to be finite sum of exponentials with generic frequencies. For small $\varepsilon$ a strong stability is shown. On the other hand, examples (in the finite difference case) are given, for which a transition in the type of spectrum occurs, as $\varepsilon$ is increased.
\end{abstract}

\section{Introduction}

Not much is known about the spectral analysis of operators of the type $H=-\Delta$ $+V$ (where $\Delta$ is the Laplacian and $V$ is some almost periodic potential) acting on $L^{2}\left(\mathbf{R}^{v}\right)$ or $l^{2}\left(\mathbf{Z}^{v}\right)$. The spectral analysis of such operators in the case of $\mathbf{R}^{1}$ could be relevant in understanding the rings of Saturn (Avron-Simon [2]) and is also of interest in insulator-conductor models of materials, Aubry [1]. The case of a random potential is of considerable interest in physics (see Thouless [10]). In any case the spectral behavior of the continuous and discrete cases are believed to be similar, and we will discuss various aspects of both of them.

In spectral analysis there are two basic questions which are of interest.

(1) What is the spectrum as a set in the plane? (We denote this set by $\sigma(H)$.)

(2) What is the nature of the eigenstates? More precisely are the eigenstates localized (or pure point spectrum) or extended (continuous spectrum) or some mixture? This latter question is the one which determines the insulator-conductor properties of the material.

We will consider more generally

$$
H_{\varepsilon}=-\Delta+\varepsilon V
$$

and examine how the spectrum depends on $\varepsilon$. The coupling constant $\varepsilon$ would correspond to the reciprocal of the temperature, in the physical models. One of the results of this paper is to give some examples of almost periodic $V$ for which the spectrum exhibits a phase transition from extended to localized states.

* Research supported in part by grant NSF MCS 7900813 
The kind of potentials we wish to discuss are of the form

and

$$
V(x)=\sum_{j=1}^{N} a_{j} e^{2 \pi i\left\langle x, \xi_{j}\right\rangle}, \quad \xi_{1}, \xi_{2}, \ldots, \xi_{N} \in \mathbf{R}^{v}
$$

$$
x \in \mathbf{R}^{v} \quad \text { or } \quad \mathbf{Z}^{v}
$$

(continuous or discrete case, respectively).

In case $V$ is periodic, then it is well known through the theory of "Bloch waves" that $\sigma\left(H_{\varepsilon}\right)$ consists of bands, and that the spectrum is absolutely continuous independent of $\varepsilon$ ! (for a full account of this see Reed-Simon [8]). In Sect. 1 we will give a derivation of these facts about the periodic case (not necessarily self adjoint) of a lattice. This is done in order to motivate various constructions in the nonperiodic case.

As far as almost periodic potentials go, some progress has been made in one dimension. Moser, Avron-Simon [6,3] have shown that for the generic limit periodic real potential the set $\sigma(H)$ is a Cantor set. Thus complicated behavior is to be expected. Sinai and Dinaburg [9] have proven that for a real quasiperiodic potential whose frequency base satisfies some generic diophantine inequalities (the continuous case), there will always be some continuous spectrum. We also mention that in dimension one Goldstein, Molchanov, Pastur [4] proved that for a random potential the spectrum is pure point (i.e. localized) with probability unity.

We will develop some theory for a generic $V(x)$ of the type 0.2 . That is to say that the $\xi_{1}, \ldots, \xi_{N}$ are chosen generically (in every sense - by Lebesgue measure or Baire category) out of $\mathbf{R}^{v}$. Of course in this case $V$ need not be real and $H$ not selfadjoint, but in view of the fact that the analysis is rather delicate, and the problem is one of "small divisors," this is a natural starting point. Indeed this is precisely the route that the classical small divisor problem of celestial mechanics took, when Siegel first overcame the small divisors in his "model problem" which was not Hamiltonian. It was only later that Kolmogorov-Arnold-Moser (KAM) were able to do the Hamiltonian case (see Moser [7]).

Since the problems we are considering are not self-adjoint, we do not look at the finer structure of the spectrum but only the question of existence or nonexistence of extended and bound states. We prove in Sect. 2 that if $\xi_{1}, \ldots, \xi_{N}$ lie inside a cone of angle less than $\pi$ (continuous case) then $\sigma\left(H_{\varepsilon}\right)=[0, \infty)$, there is no point spectrum, and the spectrum contains extended states.

If we allow any generic frequencies, for example in dimension $v=1$, $H=-\frac{d^{2}}{d x^{2}}+a e^{i \alpha x}+b e^{-i \beta x}, \alpha, \beta>0, \alpha / \beta$ generic (in fact $\alpha / \beta$ very strongly independent - see $(2.23)$ - will do), then we show that $\sigma(H)=[0, \infty)$, there is no point spectrum and there are extended states, provided that $\max \{|a|,|b|\}<\beta^{2} / 8 e^{2}$. Notice that if $a=b, \alpha=\beta$, this is the Mathieu equation which has infinitely many gaps in the spectrum. The above shows that for any neighborhood in the frequencies and any generic point there of, all gaps are closed.

More generally if

$$
H=-\frac{d^{2}}{d x^{2}}+\sum_{k=1}^{n} a_{j} e^{i \xi_{j} x}
$$


with $\left(\xi_{1}, \ldots, \xi_{n}\right)$ satisfying the generic condition of being strongly independent over $Q$ (see Definition 2.15), then we show that $\sigma(H)=[0, \infty)$ and there are extended states for $\sum_{j=1}^{n}\left|a_{j}\right|$ sufficiently small. Simple explicit examples of $\operatorname{such}\left(\xi_{1}, \ldots, \xi_{n}\right)$ are

In Sect. 3 we make a careful study of the simplest almost periodic potential on $l^{2}\left(\mathbf{Z}^{v}\right)$ :

$$
V(m)=\exp \{2 \pi i\langle m, \xi\rangle\}, \quad m \in \mathbf{Z}^{v}, \quad \xi \in \mathbf{R}^{v} \quad \text { (generic). }
$$

The following phase transition appears.

Theorem 0.3. There are constants $a_{v}<b_{v}, v \geqq 2, a_{v}, b_{v}$ increasing sequences, $a_{1}=b_{1}=1$ such that

(i) For $\varepsilon<a_{v}, \sigma\left(H_{\varepsilon}\right)=\left[0,4^{v}\right]$, there is no point spectrum, and there are extended states.

Fig. 1

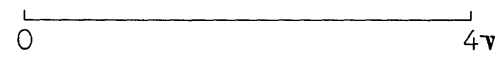

(ii) If $v>2$, and $a_{v}<\varepsilon<b_{v}$, then $\sigma\left(H_{\varepsilon}\right)$ looks like

Fig. 2

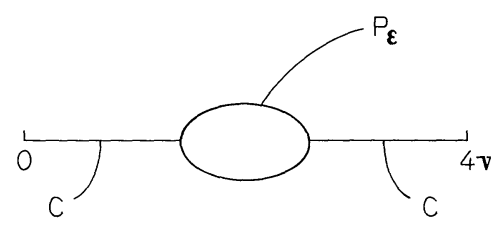

$P_{\varepsilon}$ is dense with localized states, while $C$ has extended states but no bound states. (iii) $\varepsilon>b_{v}$.

Fig. 3

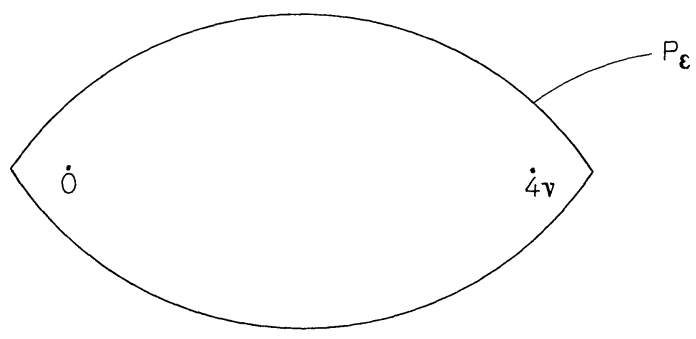

$\sigma\left(H_{\varepsilon}\right)=P_{\varepsilon}$, and $P_{\varepsilon}$ is dense with localized states.

The numbers $a_{v}, b_{v}$ are calculable, and the set $P_{\varepsilon}$ which is generally a lemniscate is actually an ellipse in one dimension.

The various strong independence or generic conditions on the coefficients cannot be dropped, without being replaced by some similar conditions. For example Gordon [13] gives examples of almost periodic, nonperiodic $V$ 's which behave very much like the periodic $V$ 's in that $(0.1)$ has no point spectrum for any $0 \leqq \varepsilon<\infty$. The frequencies he uses have the property of being very well approximable by rationals. 


\section{Section 1}

For later reference and since some of the later ideas are based on the periodic case, we derive the spectral theory for periodic potentials on $l^{2}\left(\mathbf{Z}^{v}\right)$ in this section. Since it is no harder to work with quite a general operator we do so.

Fix $v \geqq 1$, an integer, and let $\mathbf{Z}^{v}$ be the $v$ dimensional integer lattice. Let $\tilde{H}$ be a constant coefficient "partial difference operator" acting on functions on $\mathbf{Z}^{v}$. More precisely, let $D_{1}, D_{2}, \ldots, D_{v}$ be the operators

$$
D_{j} f(m)=f\left(m+e_{j}\right)-f(m), \quad m \in \mathbf{Z}^{v}
$$

and $e_{j}=(0,0,1,0, \ldots, 0)$, where 1 is in the $j^{\text {th }}$ position. Thus $\hat{H}$ is of the form $P\left(D_{1}, \ldots, D_{v}\right)$ where $P$ is a polynomial of $v$ variables.

It is plain that $\bar{H}$ is a bounded operator on $l^{2}\left(\mathbf{Z}^{v}\right)$. Further its spectral properties are easy to determine by use of the Fourier transform (or Fourier series in this case). Indeed forming the corresponding Fourier series yields a multiplication operator on $L^{2}\left(\pi^{v}\right)$ where $\pi^{v}$ denotes the $v$-torus.

$$
\tilde{H} \hat{f}\left(\theta_{1}, \ldots, \theta_{v}\right)=p\left(\theta_{1}, \ldots, \theta_{v}\right) \hat{f}\left(\theta_{1}, \ldots, \theta_{v}\right),
$$

where $p\left(\theta_{1}, \ldots, \theta_{v}\right)$ is a trigonometric polynomial. The spectrum of $\tilde{H}$ is simply the image of $\pi^{v}$ under $p$. If $\tilde{H}$ is nontrivial so that $p$ is not a constant, then clearly there is no point spectrum. If $H$ is self-adjoint then $p$ is real-valued and the spectrum is an interval.

The operators $H_{\varepsilon}$ are of the form

$$
H_{\varepsilon}=\tilde{H}+\varepsilon V,
$$

where $V$ is an almost periodic function on $\mathbf{Z}^{\nu}$.

The above paragraph concerns the case when $\varepsilon=0$. When $\varepsilon=\infty$, which we may normalize as

$$
\bar{H}_{\eta}=\eta \tilde{H}+V, \quad \eta=0,
$$

$\bar{H}_{0}$ is already a multiplication operator on $l^{2}\left(\mathbf{Z}^{v}\right)$ and is diagonal. The spectrum is pure point with the eigenfunctions being

$$
g_{m}(n)= \begin{cases}1, & \text { if } n=m \\ 0, & \text { otherwise }\end{cases}
$$

and the corresponding eigenvalue is $V(m)$. The spectrum in this case is $\left\{V(m): m \in \mathbf{Z}^{v}\right\}$. Thus at the one extreme is an absolutely continuous spectrum and at the other a pure point; the problem is to understand the transition.

By a periodic potential $V$ we mean a $V(m)$ for which there is a rank $v$ subgroup of $\mathbf{Z}^{v}$, call it $\Gamma$ such that

$$
V(m+\gamma)=V(m), \quad \forall \gamma \in \Gamma .
$$

Denoting by $\Gamma^{*}$ the dual lattice to $\Gamma$, that is $\Gamma^{*}=\left\{x \in \mathbf{R}^{v}:\langle x, \gamma\rangle \in \mathbf{Z}, \forall \gamma \in \Gamma\right\}$ we can write

$$
V(m)=\sum_{j=1}^{h} a_{j} e^{2 \pi i\langle m, \xi,\rangle},
$$


where $h=\left|\mathbf{Z}^{v} / \Gamma\right|$, and $\xi_{1}, \xi_{2}, \ldots, \xi_{h}$ are members of $\Gamma^{*}$. The above is just the finite Fourier series expansion of $V$. For a further discussion of lattices and periodic potentials on $\mathbf{R}^{v}$, see Reed and Simon [8].

Let $\theta=\left(\theta_{1}, \theta_{2}, \ldots, \theta_{v}\right)$, then on taking transforms our operator $H$ becomes

$$
(H g)(\theta)=p(\theta) g(\theta)+\sum_{j=1}^{h} a_{j} g\left(\theta-\xi_{j}\right) \quad \text { on } \quad L^{2}\left(\pi^{v}\right) \text {. }
$$

The elements $0=\xi_{1}, \ldots, \xi_{h}$ as elements of $\pi^{v}$ generate a finite subgroup which we call $G$.

To each coset $\theta+G$ of $G$ in $\pi^{v}$ we form the $h \times h$ matrix

$$
M(\theta)=D(\theta)+C,
$$

where

$$
\begin{aligned}
D(\theta) & =\left(\begin{array}{llll}
p\left(\theta+\xi_{1}\right) & & \\
& p\left(\theta+\xi_{2}\right) & & \\
& & & \\
& & & p\left(\theta+\xi_{h}\right)
\end{array}\right), \\
C & =\left(\begin{array}{llll}
a_{\xi_{1}-\xi_{1}} & a_{\xi_{1}-\xi_{2}} & \ldots & a_{\xi_{1}-\xi_{n}} \\
a_{\xi_{n}-\xi_{1}} & \ldots & & a_{\xi_{n}-\xi_{n}}
\end{array}\right] .
\end{aligned}
$$

The following is the "Bloch wave" theorem.

Theorem 1. $\sigma(H)$ is the union over $\theta$ of the eigenvalues of $M(\theta)$. Or if one chooses continuous functions $\lambda_{1}(\theta), \lambda_{2}(\theta), \ldots, \lambda_{h}(\theta)$ to describe the eigenvalues of $M(\theta)$, then $\sigma(H)=\bigcup_{i=1}^{h} \lambda_{i}\left(\pi^{v}\right)$. $H$ has no point spectrum but to each $\lambda \in \sigma(H)$ corresponds a measure $\mu$ which is supported on a coset of $G$ ( $h$ points) such that

$$
H(\mu)=\lambda \mu \text {. }
$$

(It is clear that $H$ may be extended to a bounded operator on the space of measures.)

Note 1.7. The Fourier transform $\hat{\mu}(m)$ corresponds to the "Bloch waves" and are the extended states of $H$. The spectrum is a union of the $h$ connected curves $\lambda_{i}\left(\pi^{\nu}\right)$ showing the "band structure."

Proof. Consider the equation

$$
(\lambda-H) g(\theta)=\omega(\theta) .
$$

For fixed $\theta$, we consider this equation at the points $\theta+\xi_{1}, \ldots, \theta+\xi_{h}$ and define then (1.8) becomes

$$
G(\theta)=\left[\begin{array}{c}
g\left(\theta+\xi_{1}\right) \\
\vdots \\
g\left(\theta+\xi_{h}\right)
\end{array}\right] \quad \text { and } \quad W(\theta)=\left[\begin{array}{c}
w\left(\theta+\xi_{1}\right) \\
\vdots \\
w\left(\theta+\xi_{h}\right)
\end{array}\right] \text {, }
$$

$$
(\lambda-M(\theta)) G(\theta)=W(\theta)
$$

Now if $\lambda \notin \bigcup_{i=1}^{h} \lambda_{i}\left(\pi^{v}\right)$, and if $w \in L^{2}\left(\pi^{v}\right)$, then let $G(\theta)=(\lambda-M(\theta))^{-1} W(\theta)$ for each 
$\theta$; and in particular let

$$
g(\theta)=\sum_{j=1}^{h}(\lambda-M(\theta))_{i j}^{-1} w\left(\theta+\xi_{j}\right) .
$$

Then clearly $g \in L^{2}$ and $(\lambda-H) g=w$, so that $\lambda \notin \sigma(H)$. we have

On the other hand if $\lambda \in \bigcup_{i=1}^{h} \lambda_{i}\left(\pi^{v}\right)$, then for some vector $\left[\begin{array}{c}z_{1} \\ z_{2} \\ \vdots \\ z_{h}\end{array}\right] \neq 0$ and some $\theta_{0}$,

$$
M\left(\theta_{0}\right)\left(\begin{array}{c}
z_{1} \\
\vdots \\
z_{h}
\end{array}\right)=\lambda\left(\begin{array}{c}
z_{1} \\
\vdots \\
z_{h}
\end{array}\right)
$$

Let $f$ be the measure on $\pi^{v}$ given by $g=\sum_{j=1}^{h} z_{j} \delta_{\theta_{0}+\xi_{j}}$, where $\delta$ is the point measure at zero. It is apparent that $H(g)=\lambda g$. It also follows easily by smoothing $g$ that $\lambda \in \sigma(H)$, since if $\phi_{\varepsilon}$ is an approximate identity then

$$
\phi_{\varepsilon} * g
$$

is an approximate eigenfunction.

Finally if $f \in L^{2}\left(\pi^{v}\right)$ with $H f(\theta)=\lambda f(\theta)$ for almost all $\theta$, then clearly

$$
|\lambda-M(\theta)|=0
$$

on a set of positive measure (in $\theta$ ). But this is impossible since the above determinant is a trigonometric polynomial in $\theta$ which is nonconstant. The latter follows by examination of the highest frequency which is obtained by the product of the terms down the main diagonal. This completes the proof of Theorem 1.

We learn that if $V$ is periodic, then (1.1) has no point spectrum for $0 \leqq \varepsilon<\infty$. In fact one can show the spectrum is absolutely continuous and that the same holds for the continuum $\mathbf{R}^{v}$ case, Reed, Simon [8].

Example 1.10. If $v=1, V(x)=e^{i \pi x}, x \in \mathbf{Z}, V(x+2)=V(x), \xi_{1}=0, \xi_{2}=\pi$ and take $P(D)=\Delta=$ Laplacian (nearest neighbor), then

and

$$
p(\theta)=2-2 \cos \theta,
$$

$$
\begin{aligned}
M(\theta) & =\left[\begin{array}{cc}
2-2 \cos \theta & 1 \\
1 & 2-2 \cos (\theta+\pi)
\end{array}\right] \\
\lambda_{1,2}(\theta) & =2 \pm \sqrt{1+4 \cos ^{2} \theta}
\end{aligned}
$$

There are two bands:

Fig. 4
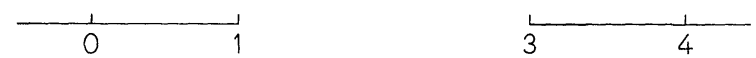


\section{Section 2}

Motivated by the construction of the extended states in the periodic case, we now try to do so in the quasi-periodic "generic potential" case.

For simplicity we now restrict ourselves to

$$
H_{\varepsilon}=-\Delta+\varepsilon V
$$

though what we do in this section would work if $\Delta$ were replaced by any constant coefficient elliptic operator.

For the following discussion, assume we are in $\mathbf{R}^{v}$ or $\mathbf{Z}^{v}$, whose variable we denote by $x$, and dual variable by $\xi$.

$$
V(x)=\sum_{j=1}^{N} a_{j} e^{2 \pi i\left\langle x, \xi_{j}\right\rangle} .
$$

On taking transforms in (2.1) we obtain a spectral equation just as before:

where

$$
P_{\lambda}(\xi) f(\xi)=\sum_{j=1}^{N} a_{j} f\left(\xi+\xi_{j}\right)
$$

$$
P_{\lambda}(\xi)= \begin{cases}\lambda-|\xi|^{2} & \text { for } \mathbf{R}^{v} \\ \lambda-\left(2 v-2 \sum_{k=1}^{v}\left(\cos \xi_{j}\right)\right) & \text { for } \mathbf{Z}^{v}\end{cases}
$$

Suppose that $\xi_{0}$ is chosen so that

$$
p_{\lambda}\left(\xi_{0}\right)=0 \text {. }
$$

We try to construct a measure $\mu$ supported on the orbit through $\xi_{0}$, under $\mathbf{Z}^{N}$ action:

$$
\left(s_{1}, s_{2}, \ldots, s_{N}\right) \rightarrow \xi_{0}+s_{1} \xi_{1}+s_{2} \xi_{2}+\ldots+s_{N} \xi_{N}
$$

So $\mu$ is of the form

$$
\mu=\sum_{s_{1}, s_{2}, \ldots, s_{N} \in \mathbf{Z}^{N}} \gamma_{s_{1}, s_{2}, \ldots, s_{N}} \delta_{\xi_{0}+s_{1} \xi_{1}+\ldots+s_{N} \xi_{N}}(\xi) .
$$

Then

$$
\begin{aligned}
\mu\left(\xi+\xi_{j}\right) & =\sum_{s} \gamma_{s} \delta_{\xi_{0}+s_{1} \xi_{1}+\ldots+s_{N} \xi_{N}}\left(\xi+\xi_{j}\right) \\
& =\sum_{s} \gamma_{s} \delta_{\xi_{0}+s_{1} \xi_{1}+\ldots+\left(s_{j}-1\right) \xi_{j}+\ldots+s_{N} \xi_{N}}(\xi) \\
& =\sum_{s} \gamma_{s-e_{j}} \delta_{\xi_{0}+s_{1}} \xi_{1}+\ldots+s_{N} \xi_{N}(\xi)
\end{aligned}
$$

where $e_{j}=(0, \ldots, 1,0, \ldots, 0)$, and where 1 is in the $j^{\text {th }}$ position.

Now (2.2) leads to

$$
\sum_{j=1}^{N} a_{j} \gamma_{s-e_{j}}=p_{\lambda}\left(\xi_{0}+s_{1} \xi_{1}+\ldots+s_{N} \xi_{N}\right) \cdot \gamma_{s}
$$


If we can solve for the coefficients $\gamma_{s}, s \in \mathbf{Z}^{N}$, and also have

$$
\sum_{s \in \mathbf{Z}^{N}}\left|\gamma_{s}\right|<\infty
$$

then $H \mu=\lambda \mu$, and $\hat{\mu}(x)$ will be an extended state.

The system (2.5) is just a first order hyperbolic system (finite difference) on the $\mathbf{Z}^{N}$ lattice, and one may formally solve it by the method of characteristics. Since we have chosen

$$
P\left(\xi_{0}\right)=0 \text {, }
$$

we see that we may introduce a "source" at $(0,0, \ldots, 0) \in \mathbf{Z}^{N}$ by setting

and

$$
\gamma_{s}=0 \quad \text { if } \quad s_{i}<0 \text { for some } i
$$

$$
\gamma_{0}=1
$$

We have satisfied (2.5) except in the first "quadrant." Now proceed inductively to solve for $\gamma_{s}$ on the hyperplanes $s_{i} \geqq 0, s_{1}+s_{2}+\ldots+s_{N}=m, m=1,2, \ldots$, which can be done by use of (2.5) as long as

$$
p_{\lambda}\left(\xi_{0}+s_{1} \xi_{1}+\ldots+s_{N} \xi_{N}\right) \neq 0 \quad \text { for } \quad s_{i} \geqq 0, s \neq 0 .
$$

To illustrate the above, take $N=2$



A little closer look reveals that we may write $\gamma_{s}$ as a sum over all paths joining 0 to $s$, of certain products along these paths. If $s_{i} \geqq 0$,

$$
\begin{gathered}
\gamma_{s}=\sum_{\substack{e_{j_{1}}+e_{j_{2}}+\ldots+e_{j_{k}}=s \\
j_{N} \in\{1,2, \ldots, N\}}} \prod_{r=1}^{k} \frac{a_{j_{r}}}{p\left(\xi_{0}+\xi_{j_{1}}+\ldots+\xi_{j_{r}}\right)}, \\
\text { where } \quad k=s_{1}+s_{2}+\ldots+s_{N} .
\end{gathered}
$$


Thus formally subject to (2.7) we have found an "eigenmeasure" $\mu$. The question of the convergence of (2.6) depends on the behavior of the products of $p_{\lambda}$ along the paths and, of course from (2.8), is a small divisor problem. Before discussing these analytic questions we show that the same products occur in inverting

$$
H_{\varepsilon, \lambda}=\lambda-(\Delta+\varepsilon V) .
$$

Now assume that $p_{\lambda}(\xi) \neq 0$ for any $\xi$. Let $-\hat{w} \in L^{2}\left(\mathbf{R}^{v}\right)\left(\right.$ or $\left.l^{2}\left(\mathbf{Z}^{v}\right)\right)$, then if we are to show that $\lambda \notin \sigma(H)$ we need to solve

$$
(\lambda-(\Delta+V)) \hat{f}=-\hat{w}, \quad \hat{f \in} L^{2} .
$$

This leads to

or

$$
-p_{\lambda}(\xi) f(\xi)+\sum_{j=1}^{N} a_{j} f\left(\xi+\xi_{j}\right)=-w(\xi),
$$

$$
f(\xi)=\frac{1}{p_{\lambda}(\xi)}\left\{w(\xi)+\sum_{j=1}^{N} a_{j} f\left(\xi+\xi_{j}\right)\right\} .
$$

We now substitute this expression for $f(\xi)$ repeatedly back into the right hand side. Doing this $k$ times yields:

$$
\begin{aligned}
f(\xi)= & \frac{w(\xi)}{p_{\lambda}(\xi)}+\sum_{j_{1}=1}^{N} \frac{a_{j_{1}} w\left(\xi+\xi_{j_{1}}\right)}{p_{\lambda}(\xi) p_{\lambda}\left(\xi+\xi_{j_{1}}\right)}+\ldots \\
& +\sum_{j_{1}=1}^{N} \sum_{j_{2}=1}^{N} \ldots \sum_{j_{k-1}=1}^{N} \frac{a_{j_{1}} \ldots a_{j_{k-1}} w\left(\xi+\xi_{j_{1}}+\ldots+\xi_{j_{k-1}}\right)}{p_{\lambda}(\xi) p_{\lambda}\left(\xi+\xi_{j_{1}}\right) \ldots p_{\lambda}\left(\xi+\xi_{j_{1}}+\xi_{j_{k-1}}\right)} \\
& +\sum_{j_{1}=1}^{N} \sum_{j_{2}=1}^{N} \ldots \sum_{j_{k}=1}^{N} \frac{a_{j_{1}} \ldots a_{j_{k}} f\left(\xi+\xi_{j_{1}}+\ldots+\xi_{j_{k}}\right)}{p_{\lambda}\left(\xi+\xi_{j_{1}}\right) \ldots p_{\lambda}\left(\xi+\xi_{j_{1}}+\ldots+\xi_{j_{k}}\right)} .
\end{aligned}
$$

We see that the same products over the same paths control the terms in the above series. If we can let $k \rightarrow \infty$ so that the series converges, then the above would express $f(\xi)$ in terms of $w(\xi)$.

This completes the formal aspect; we now turn to questions of convergence, and hence the analysis of the products of $p_{\lambda}$ along such paths. We will do so by giving examples where we have been able to make the necessary analytic estimates.

Example. (i) Take the example of $\mathbf{R}^{v}$, and such that the frequencies $\xi_{1}, \ldots, \xi_{N}$ lie in a set $A$ which has the following property:

If $A^{(n)}=A+A+\ldots+A n$ times, then we want

$$
\begin{gathered}
\quad d\left(0, A^{(n)}\right) \geqq c n \quad \text { for large } n, \\
\text { where } \quad d(x, y)=\text { distance from } x \text { to } y .
\end{gathered}
$$

The condition (2.11) is easily met if, say, $\xi_{1}, \ldots, \xi_{N}$ lie in a cone in $\mathbf{R}^{v}$ with angle less than $\pi$, or if $N \leqq v$ and $\xi_{1}, \ldots, \xi_{N}$ are linearly independent.

Theorem 2.12. If $\xi_{1}, \ldots, \xi_{N} \in A$ and $A$ satisfies (2.11) then

and $H$ has no point spectrum.

$$
\sigma(H)=[0, \infty)
$$


Proof. The $a_{1}, \ldots, a_{N}$ are fixed. First, we show that if $\lambda \notin[0, \infty)$ then $\lambda-H$ is invertible. In this case, fix $\lambda$;

$$
\begin{gathered}
p_{\lambda}(\xi)=\lambda-|\xi|^{2} \neq 0 \quad \text { for all } \quad \xi \in \mathbf{R}^{v}, \\
\left|p_{\lambda}\left(\xi+\xi_{j_{1}}+\xi_{J_{2}}+\ldots+\xi_{j_{n}}\right)\right|=\left.|\lambda-|\left(\xi+\xi_{j_{1}}+\ldots+\xi_{j_{n}}\right)\right|^{2} \mid
\end{gathered}
$$

so for large $m$ it is clear what the behavior is, subject to (2.11). Hence:

$$
\left|\frac{1}{p_{\lambda}\left(\xi+\xi_{j_{1}}+\ldots+\xi_{m}\right)}\right| \leqq \frac{C(\xi)}{m^{2}}
$$

for a suitable function $C(\xi)$ on $\mathbf{R}^{v}$.

Now for a typical term in the series (2.10) we have

$$
\begin{aligned}
& \left\|\frac{a_{j_{1}} \ldots a_{j_{k}} w\left(\xi+\xi_{j_{1}}+\ldots+\xi_{j_{k-1}}\right)}{p_{\lambda}(\xi) \ldots p_{\lambda}\left(\xi+\xi_{j_{1}}+\ldots+\xi_{j_{k-1}}\right)}\right\|_{2} \\
\leqq & \|w\|_{2}(B)^{k}\|C(\xi)\|_{\infty} \\
(k !)^{2} & \text { for some } B .
\end{aligned}
$$

Thus if we can show that $C \in L^{\infty}$, then letting $k \rightarrow \infty$ gives us $f \in L^{2}$. But it is easy to see that $C$ is in $L^{\infty}$ since it certainly can be chosen to be continuous as well as chosen so that $C(\xi) \rightarrow 0$ at infinity. Indeed, one has some $\varepsilon>0$ such that

$$
\left|p_{\lambda}(\xi)\right| \geqq \varepsilon, \quad \forall \xi,
$$

while the number of steps that our path can spend in a region where $\left|p_{\lambda}(\xi)\right| \leqq 1$ is bounded, so the above follows.

At this point we have shown that $\sigma(H) \subset[0, \infty)$ : To see there is no point spectrum, suppose $\lambda \in[0, \infty)$ with $H f=\lambda f, f \in L^{2}$. Then (2.10) will still hold for those $\xi$ for which $p_{\lambda}\left(\xi+\xi_{j_{1}}+\ldots+\xi_{j_{k}}\right) \neq 0$ for any $k$. This set is clearly of full measure in $\mathbf{R}^{v}$.

For such $\xi$ and any $m$ we have

$$
f(\xi)=\sum_{j_{1}=1}^{N} \sum_{\ldots} \sum_{j_{m}=1}^{N} \frac{a_{j_{1}} \ldots a_{j_{m}} f\left(\xi+\xi_{j_{1}}+\ldots+\xi_{j_{m}}\right)}{p_{\lambda}(\xi) p_{\lambda}\left(\xi+\xi_{j_{1}}\right) \ldots p_{\lambda}\left(\xi+\xi_{j_{1}}+\ldots+\xi_{j_{m}}\right)} .
$$

But for such $\xi$ (2.12) holds for large $m$ so that taking the limit as $m \rightarrow \infty$ yields

$$
f(\xi)=0 \quad \text { a.e. }
$$

i.e. there is no point spectrum.

Finally if $\lambda \in[0, \infty)$ we construct an extended state as described earlier. One can for most $\lambda$ 's construct this with multiplicity of the ${ }^{v}$ sphere, but the simplest is to choose $\xi_{0}$ with $\left|\xi_{0}\right|^{2}=\lambda$ and such that $\xi_{0}$ is in the same cone as $A$ (e.g. choose $\xi_{0}$ to point in the direction of any element of $A$ ). Doing this we have $p_{\lambda}\left(\xi_{0}\right)=0$ as needed for (2.3), and $p_{\lambda}\left(\xi_{0}+\xi_{j_{1}}+\ldots+\xi_{j_{k}}\right)$ is bounded away from zero, so again (2.12) comes into force with $\xi=\xi_{0}$, from which the c:Invergence of the series (2.6) follows.

This completes the proof of (2.12). 
Example. (ii) We turn to a more interesting example. In dimension $v=1$ consider

$$
H=-\frac{d^{2}}{d x^{2}}+V(x)
$$

where

$$
V(x)=\sum_{j=1}^{n} a_{j} e^{i \xi_{j} x} .
$$

The point $\left(\xi_{1}, \ldots, \xi_{n}\right) \in \mathbf{R}^{n}$ will be described in more detail shortly and will be generic.

Before continuing we need some definitions and estimates concerning certain sequences.

Definition 2.15. A point $\xi=\left(\xi_{1}, \ldots, \xi_{n}\right) \in \mathbf{R}^{n}$ will be called strongly independent over $\mathbf{Z}$ with parameters $(c, \beta)$, where $c, \beta>0$ if

$$
\left|m_{1} \xi_{1}+\ldots+m_{n} \xi_{n}\right| \geqq C|m|^{-\beta} \quad \text { for } \quad 0 \neq m=\left(m_{1}, \ldots, m_{n}\right) \in \mathbf{Z}^{n} .
$$

It is not difficult to show that $\left\{\xi \in \mathbf{R}^{n}: \exists c_{\xi}, \beta_{\xi}>0\right.$ for which (2.15) holds $\}$ is a set of full measure. Thus the condition (2.15) is generic. To obtain explicit examples one can use algebraic number theory. For example if $K / Q$ is a real extension of degree $n$, and if $\xi_{1}, \ldots, \xi_{n}$ is a $\mathbf{Z}$ basis for $K$, then (2.15) holds with suitable $C$ and $\beta=n-1$ (see, for example, Meyer [12]).

We now introduce a useful function which appears in Katznelson [11]. Let

$$
N(u)=\inf _{\substack{m_{i} \geq 0 \\ 0 \neq m \in \mathbf{Z}^{n}}}\left\{m_{1}+m_{2}+\ldots+m_{n}|| m_{1} \xi_{1}+\ldots+m_{n} \xi_{n} \mid<u\right\} .
$$

It follows that if $\xi_{1}, \ldots, \xi_{n}$ are strongly independent over $Q$ with parameters $(c, \beta)$ then

$$
N(u) \geqq C^{1 / \beta} u^{-1 / \beta} .
$$

By previous remarks it is clear that an estimate like (2.16) is true for generic $\left(\xi_{1}, \ldots, \xi_{n}\right)$.

Let $F=\left\{\xi_{1}, \ldots, \xi_{n}\right\}$. By $\left\{x_{1}, \theta\right\}_{i=0}^{k}$ being an $F$-sequence, we mean that $x_{i+1}-x_{i} \in F$ and $x_{0}=\theta$. From (2.8) and (2.10) it is clear that the arguments of the $p_{\lambda}$ 's are $F$ sequences.

Proposition 2.18. If $\left(\xi_{1}, \ldots, \xi_{n}\right) \in \mathbf{R}^{n}$ is strongly independent with parameters $(c, \beta)$, then for any $F$ sequence $\left\{x_{i}, 0\right\}_{i=0}^{k}$ we have

$$
\left(\prod_{i=1}^{k}\left|x_{i}\right|\right)^{1 / k} \geqq \exp \left(-\beta\left(\frac{2}{c}\right)^{1 / \beta}\right)
$$

This proposition tells us that strongly independent $F$-sequences have absolute lower bounds for their geometric means.

Proof. Fix $k$ and an $F$ sequence $x_{1}, \ldots, x_{k}$. Let

$$
N_{k}(u)=\#\left\{x_{j}:\left|x_{j}\right|<u\right\} .
$$


Now

$$
\begin{aligned}
\log \left(\prod_{i=1}^{k}\left|x_{i}\right|\right)^{1 / k} & =\frac{1}{k} \sum_{i=1}^{k} \log \left|x_{i}\right|=\frac{1}{k} \int_{0}^{\infty} \log u d N_{k}(u) \\
& =\frac{1}{k} \int_{0}^{1} \log u d N_{k}(u) \geqq-\frac{1}{k} \int_{0}^{1} \frac{N_{k}(u)}{u} d u
\end{aligned}
$$

Now clearly

$$
\begin{aligned}
\frac{N_{k}(u)}{N(a u)+\ldots+N(a u)} \leqq k \\
N_{k}(u) \leqq \frac{k}{N(a u)} \leqq \frac{k}{C^{1 / \beta}}(2 u)^{1 / \beta} \\
\frac{1}{k} \int_{0}^{1} \frac{N_{k}(u)}{u} d u \leqq \beta\left(\frac{2}{C}\right)^{1 / \beta}, \\
\left(\prod_{i=1}^{k}\left|x_{i}\right|\right)^{1 / k} \geqq \exp \left(-\beta\left(\frac{2}{c}\right)^{1 / \beta}\right) .
\end{aligned}
$$

Proposition 2.19. Let $\omega=\omega_{1}+i \omega_{2} \in \mathbf{C}, \omega_{2} \neq 0$ and $\theta \in \mathbf{R}$, then for any $F$ sequence $\left\{x_{i}, \theta\right\}_{i=0}^{k}, F$ as above

$$
\left(\prod_{i=1}^{k}\left|x_{i}-\omega\right|\right)^{1 / k} \geqq C_{\omega_{2}}^{1 / k} \exp \left(-\beta\left(\frac{2}{c}\right)^{1 / \beta}\right),
$$

where $C_{\omega_{2}}>0$ and depends on $\omega_{2}$ only.

Proof.

$$
\cdot w_{1}+i w_{2}
$$

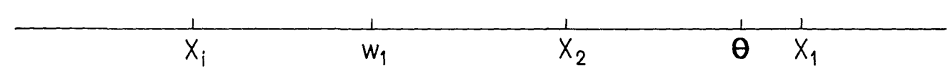

Fig. 6

We have $\frac{1}{k} \sum_{i=1}^{k} \log \left|x_{i}-\omega\right| \geqq \frac{1}{k} \int_{0}^{1} \log \left|\omega_{1}-\left(u+\omega_{1}\right)\right| d N_{k}(\omega, u, \theta)$,

where $N_{k}(\omega, u, \theta)=\#\left\{x_{i}:\left|x_{i}-\omega_{1}\right| \leqq u\right\}$;

$$
\frac{1}{k} \sum_{i=1}^{k} \log \left|x_{i}-\omega\right| \geqq-\frac{1}{k} \int_{0}^{1} \frac{1}{\sqrt{\omega_{2}^{2}+u^{2}}} N_{k}(\omega, u, \theta) d u .
$$

Now it is clear that independent of $\theta$ we have

$$
\begin{aligned}
\frac{N_{k}(\omega, u, \theta)-1 \text { times }}{N(a u)+\ldots+N(a u)} & \leqq k, \\
N_{k}(\omega, u, \theta) & \leqq \frac{k}{N(a u)}+1, \\
\frac{1}{k} \int_{0}^{1} \frac{1}{\sqrt{\omega_{2}^{2}+u^{2}}} N_{k}(\omega, u, \theta) d u & \leqq\left(\frac{2}{c}\right)^{1 / \beta} \beta+\frac{1}{k} \int_{0}^{1} \frac{1}{\left|\omega_{2}\right|} d u,
\end{aligned}
$$

which proves the result. 
Now suppose $\left\{\xi_{1}, \ldots, \xi_{n}, \alpha_{1}, \alpha_{2}\right\}$ is strongly independent over $Q$ with parameters $(C, \beta)$. Of cerurse the set of such $\left(\xi_{1}, \ldots, \xi_{n}\right) \in \mathbf{R}^{n}$ is still generic, and the set of numbers $m \alpha_{1}+n \alpha_{2}, m, n \in \mathbf{Z}$ is dense in $\mathbf{R}$.

Theorem 2.20. If $\xi_{1}, \ldots, \xi_{n}$ are as above and

$$
H=-\frac{d^{2}}{d x^{2}}+\sum_{j=1}^{n} a_{j} e^{i \xi_{j} x}
$$

then for $\max \left\{\left|a_{1}\right|, \ldots,\left|a_{n}\right|\right\}<\exp \left\{-2 \beta\left(\frac{2}{c}\right)^{1 / \beta}\right\} / n$ we have $\sigma(H)=[0, \infty)$.

Remarks. (1) The theorem as stated does not allow for a zero frequency, that is a term $a_{0}$, however such a term will simply shift the spectrum.

(2) Concrete examples for which the theorem holds can be written down by use of the remarks following (2.15). For example:

$$
\begin{aligned}
H= & -\frac{d^{2}}{d x^{2}}+a_{1} e^{i \sqrt{2} x}+a_{2} e^{-i \sqrt{3} x}+a_{3} e^{i \sqrt{7} x}+a_{4} e^{-i \sqrt{21} x} \\
& +a_{5} e^{i \sqrt{6} x}+a_{6} e^{i \sqrt{14} x}
\end{aligned}
$$

will satisfy $\sigma(H)=[0, \infty)$ if

$$
\max _{i=1, \ldots, 6}\left\{\left|a_{i}\right|\right\} \leqq 10^{-8} .
$$

This follows from considerations in $Q(\sqrt{2}, \sqrt{3}, \sqrt{7})$.

Proof of (2.20). First choose $\lambda \notin[0, \infty)$, then we have the following lower bound for the product of the $p_{\lambda}$ 's along a path of length $k$,

$$
\begin{aligned}
& \left|p_{\lambda}(\theta) p_{\lambda}\left(\theta+\xi_{j_{1}}\right) \ldots p_{\lambda}\left(\theta+\xi_{j_{k}}\right)\right| \\
& =|\theta+\sqrt{\lambda}|\left|\theta+\xi_{j_{1}}+\sqrt{\lambda}\right| \ldots\left|\theta+\xi_{j_{1}}+\ldots+\xi_{j_{k}}+\sqrt{\lambda}\right| \\
& \quad \times|\theta-\sqrt{\lambda}|\left|\theta+\xi_{j_{1}}-\sqrt{\lambda}\right|+\ldots+\left|\theta+\xi_{j_{1}}+\ldots+\xi_{j_{k}}-\sqrt{\lambda}\right| \\
& \geqq C_{\lambda}\left(\exp \left\{-\left(\frac{2}{c}\right)^{1 / \beta} \beta\right\}\right)^{2 k},
\end{aligned}
$$

where $c_{\lambda}=c(\lambda)>0$, by $(2.18)$, since $\pm \sqrt{\lambda} \notin[0, \infty)$. It follows as before that if

$$
\max \left\{\left|a_{1}\right|, \ldots,\left|a_{n}\right|\right\}<\frac{e^{-2 \beta(2 / c)^{1 / \beta}}}{n}
$$

the series (2.10) converges, and thus $\lambda \notin \sigma(H)$.

On the other hand if $\lambda \in[0, \infty) \cap\left\{m \alpha_{1}+n \alpha_{2}: m, n \in \mathbf{Z}\right\}$ which is dense in $[0, \infty)$ we will show that $\lambda \in \sigma(H)$. This will complete the proof of the theorem. 
For such a $\lambda=m \alpha_{1}+n \alpha_{2}$ choose $\xi_{0} \geqq 0, \xi_{0}^{2}=\lambda$, and so $p_{\lambda}\left(\xi_{0}\right)=0$. Construct $\mu$ as in (2.4). Now

$$
\begin{aligned}
& \prod_{r=1}^{k} p_{\lambda}\left(\xi_{0}+\xi_{j 1}+\ldots+\xi_{j r}\right) \\
& \quad=\left(\prod_{r=1}^{k}\left|\xi_{j 1}+\ldots+\xi_{j r}\right|\right) \times\left(\prod_{r=1}^{k}\left|2 \xi_{0}+\xi_{j 1}+\ldots+\xi_{j r}\right|\right) \\
& \quad=\left(\prod_{r=1}^{k}\left|\xi_{j 1}+\ldots+\xi_{j r}\right|\right)\left(\prod_{r=1}^{k}\left|2 m \alpha_{1}+2 n \alpha_{2}+\xi_{j 1}+\ldots+\xi_{j r}\right|\right) \\
& \quad \geqq C_{m, n}\left(\exp \left\{-\left(\frac{2}{c}\right)^{1 / \beta} \beta\right\}\right)^{k}\left(\exp \left\{-\left(\frac{2}{c}\right)^{1 / \beta} \beta\right\}\right)^{k},
\end{aligned}
$$

with $C_{m, n}>0$, by Proposition 2.17.

It follows that the series $\Sigma\left|\gamma_{s}\right|$ of (2.8) is finite if the condition (2.21) is satisfied. Thus by previous arguments $\hat{\mu}$ is an extended state and $\lambda \in \sigma(H)$.

If there are only two frequencies $\alpha, \beta$ which are strongly independent over $Q$, then we can make a slightly stronger statement.

\section{Theorem 2.22.}

$$
H=-\frac{d^{2}}{d x^{2}}+a e^{i \alpha x}+b e^{i \beta x}, \quad \alpha>0, \quad \beta<0,
$$

then if $\max \{|\mathrm{a}|,|b|\}<\beta^{2} / 8 e^{2}$ (one could choose to make $\alpha$ more important as well), then $\sigma(H)=[0, \infty)$ and $H$ has no point spectrum. ( $e=$ natural base of logarithms.)

The proof is very similar to that of (2.20); the improvement comes from a slightly better method of estimating the products of $p_{\lambda}$ along paths. Before outlining this proof we state two very useful lemmas, whose proofs are in the appendix.

Definition 2.23. $\left(\xi_{1}, \ldots, \xi_{n}\right) \in \mathbf{R}^{n}$ is very strongly independent over $Q$ if $\exists c, \sigma>0$ such that

$$
\left\|m_{1} \xi_{1}+\ldots+m_{n} \xi_{n}\right\| \geqq c|m|^{-\sigma} \quad \text { for } \quad m \neq 0, m \in \mathbf{Z}^{v},
$$

where \|\| denotes the distance to the nearest integer. Again this condition is generic for $\xi \in \mathbf{R}^{n}$.

Lemma 2.24. Let $g(\theta)$ be smooth on $\pi^{v}$ and suppose that $g(\theta) \neq 0$ for all $\theta$, then if $\xi$ is strongly independent over $Q$ and if $\Gamma_{n}(\theta)=\prod_{r=0}^{n-1} g(\theta+r \xi)$, then

$$
\frac{1}{n} \log \left|\Gamma_{n}(\theta)\right| \rightarrow \frac{1}{(2 \pi)^{v}} \int_{\pi^{v}} \log |g(\theta)| d \theta
$$

uniformly in $\theta$, as $n \rightarrow \infty$.

Lemma 2.25. Let $\alpha \in \mathbf{R}$ be very strongly independent over $Q$ and let $r \in Q$, then for suitable constant $C_{\alpha, r}>0$,

$$
\prod_{k=1}^{n}\|k \alpha+r\| \geqq C_{\alpha, r}(2 e)^{-n}
$$


Proof of (2.22). We proceed exactly as before, but first one may normalize as follows:

$$
H=-\frac{d^{2}}{d x^{2}}+\tilde{a} e^{i \xi x}+\tilde{b} e^{-i x}, \quad \tilde{a}=\frac{a}{\beta^{2}}, \tilde{b}=\frac{b}{\beta^{2}},
$$

and $\xi=\alpha / \beta$ is very strongly independent over $Q$.

Then as before we need to estimate products like

$$
|\sqrt{\lambda}+\theta|\left|\sqrt{\lambda}+\theta+\xi_{j_{1}}\right| \ldots\left|\sqrt{\lambda}+\theta+\xi_{j_{1}}+\ldots+\xi_{j_{k}}\right|
$$

where $\sqrt{\lambda} \notin[0, \infty)$ at $\xi_{j_{r}} \in\{-1, \xi\}$. We may write this as

$$
|\sqrt{\lambda}+\theta|\left|\sqrt{\lambda}+\theta+m_{1} \xi-n_{1}\right| \ldots\left|\sqrt{\lambda}+\theta+m_{k} \xi-n_{k}\right|,
$$

where $0 \leqq m_{1} \leqq m_{2} \ldots \leqq m_{k}$ and $m_{k}-m_{k-1} \leqq 1$ is determined by the path on which we are trying to make the estimate. It is clear, since the distance between integers is greater than or equal to 1 , that

$$
|\sqrt{\lambda}+\theta| \ldots\left|\sqrt{\lambda}+\theta+m_{k} \xi-n_{k}\right| \geqq\|\sqrt{\lambda}+\theta\| \ldots\|\sqrt{\lambda}+\theta+k \xi\|,
$$

where $\|z\|$ is the distance from $z \in \mathbf{C}$ to the nearest integer.

Writing $\sqrt{\lambda}=u+i v, u, v \in \mathbf{R}, v \neq 0$ we are reduced to the study of the sequence $u+\theta+k \xi$ modulo 1 . The last product can be bounded from below by $\prod_{j=0}^{k}|g(\theta+j \xi)|$, where $g(\theta)$ is smooth on the unit circle (here it is $[0,1]$ with zero and one identified), and $g(\theta) \neq 0$ for all $\theta$, and

$$
\int_{0}^{1} \log |g(\theta)| d \theta \geqq \int_{0}^{1} \log \|u+x\| d x=-(1+\log 2) .
$$

By (2.24) we have

$$
\begin{aligned}
\left(\prod_{\substack{\text { along path } \\
\text { length } k}}\left|p_{\lambda}(\theta)\right|\right) & \geqq C\left(e^{-(1+\log 2)}\right)^{2 k} \\
& =\frac{C}{\left(4 e^{2}\right)^{k}} .
\end{aligned}
$$

It follows as before that if

$$
\max \{|\tilde{a}|,|\tilde{b}|\}<\frac{1}{8 e^{2}}
$$

then series (2.10) converges and so $\lambda \notin \sigma(H)$. Again just as in example (i), even if $\lambda \in[0, \infty)(2.26)$ will hold for a.e. $\theta(C=C(\theta))$ by the ergodic theorem, so that as before there can be no $L^{2}$ solutions of $H f=\lambda f-$ i.e. there is no point spectrum if (2.27) holds.

Finally if $\lambda \in[0, \infty) \cap Q$, then (2.25) shows that if $\xi_{0}^{2}=\lambda$, i.e. $p_{\lambda}\left(\xi_{0}\right)=0$,

$$
\left|\prod_{k=1}^{n} p_{\lambda}\left(\xi_{0}+k \xi\right)\right| \geqq C_{\xi, r}\left(4 e^{2}\right)^{-n}
$$


so that from (2.8), $\Sigma\left|\gamma_{s}\right|<\infty$ if (2.27) holds, or $\mu_{\lambda}$ as constructed in (2.4) has $\|\mu\|$ $<\infty$ and $H \mu_{\lambda}=\lambda \mu_{\lambda}$. This completes the proof.

We conclude this section by observing that a proof analogous to (2.20) can clearly be carried out for the case of the finite difference Laplacian (in dimension one), plus a potential whose frequencies are very strongly independent over $Q$.

The analogoue of (2.22) for $L^{2}\left(\mathbf{R}^{v}\right)$ can be carried out for a $V(x)$ which has at most $n+1$ frequencies.

\section{Section 3}

Thus far the behavior we have found is the same as the periodic case insofar as there is no point spectrum. We will now consider what is probably the simplest almost periodic potential and make a complete analysis of the spectral behavior as we vary the coupling constant $\varepsilon$.

We take for $V$ the function $\exp \{2 \pi i\langle m, \xi\rangle\}$ on the $\mathbf{Z}^{v}$ lattice and $\xi$ will be a generic point of $\mathbf{R}^{v}$.

$$
H_{\varepsilon}=-\Delta+\varepsilon V
$$

where as usual $\Delta$ is the finite difference Laplacian. In this case since the corresponding product of the $p_{\lambda}(\theta)$ 's along the paths is just

$$
p_{\lambda}(\theta) p_{\lambda}(\theta+\xi) \ldots p_{\lambda}(\theta+k \xi)
$$

(since there is only one frequency), we will be able to make a complete analysis. The results were already described in the introduction.

We begin by considering some equations on the torus.

3.2. Consider the equation

$$
f(\theta+\xi)-f(\theta)=g(\theta) \quad \text { on } \quad \pi^{v} ;
$$

$g$ being given and we must solve for $f$.

Clearly a necessary condition for an $L^{1}$ solution of (3.3) is that

$$
\hat{g}(0)=\frac{1}{(2 \pi)^{v}} \int_{\pi} g(\theta) d \theta=0 .
$$

If this condition is satisfied one may still have difficulty solving for $f$ if $\xi=\left(\xi_{1}, \ldots, \xi_{v}\right)$ satisfies some relations over $Q$. If

$$
\left|e^{2 \pi i\langle\xi, m\rangle}-1\right| \neq 0 \quad \text { for } \quad m \in \mathbf{Z}^{v}, \quad m \neq 0,
$$

then formally the Fourier coefficients of $f(\theta)$ are given by

$$
\hat{f}(m)=\left\{\begin{array}{ll}
0 & \text { if } m=0 \\
\hat{g}(m) /\left(e^{2 \pi i\langle m, \xi\rangle}-1\right),
\end{array} \quad m \in \mathbf{Z}^{v} .\right.
$$

To ensure that we have the coefficients of a function $f(\theta)$ one needs to impose further conditions on $g(\theta)$ and $\xi$. For example if $\xi$ is very strongly independent over $Q$ (as in (2.23)) and if $g(\theta)$ is smooth, then clearly the coefficients $\hat{f(m)}$ above are 
those of a smooth function. If $g$ is analytic in a neighborhood of $\pi^{v}$, then $f$ will also be analytic in any smaller neighborhood of $\pi^{\nu}$.

For the rest of this section assume that $\xi$ is very strongly independent.

3.5. A related equation is the following

$$
f(\theta+\xi)=g(\theta) f(\theta) \quad \text { on } \pi^{\nu} .
$$

First we remark that if $g\left(\theta_{0}\right)=0$ for some $\theta_{0}$, then iteration of the above yields

$$
f\left(\theta_{0}+n \xi\right)=0 \quad \forall n \geqq 1 .
$$

Thus since we are assuming $\xi$ to be independent over $Q$, we have by Kronecker's theorem $n \xi$ is dense in $\pi^{v}$; we see that no nontrivial continuous solution of (3.6) exists. Similarly if $f\left(\theta_{0}\right)=0$ and $f$ is continuous, then $f \equiv 0$. So if we are looking for continuous nonzero solutions of (3.6) we can assume that neither $f$ nor $g$ vanishes.

Assume a branch of $\arg g(\theta)$ is continuous; then (3.6) leads to

$$
\log f(\theta+\xi)-\log f(\theta)=\log g(\theta) .
$$

So a necessary condition to solve this is as before

$$
\int_{\pi^{v}} \log |g(\theta)| d \theta=0, \quad \int_{\pi^{v}} \arg g(\theta)=0 .
$$

Assuming $g(\theta)$ to be smooth (respectively analytic), we may find $f_{1}(\theta)$ smooth (analytic) such that

so that

$$
f_{1}(\theta+\xi)-f_{1}(\theta)=\log g(\theta)
$$

$$
f(\theta)=e^{f_{1}(\theta)}
$$

gives a smooth (analytic) solution to (3.6).

However one may solve (3.6) more generally if

$$
\left\{\begin{aligned}
\frac{1}{(2 \pi)^{v}} \int_{\pi^{v}} \arg (g(\theta)) d \theta & =2 \pi m, \quad m \in \mathbf{Z}, \quad \text { and } \\
\int \log |g| & =0
\end{aligned}\right.
$$

since then $\log (g(\theta))-2 \pi i m$, may by the above reasoning be written as $f_{1}(\theta+\xi)-f_{1}(\theta)$ and so $g(\theta)=f(\theta+\xi) / f(\theta)$, where $f=\exp \left\{f_{1}\right\}$.

Furthermore a constant $C$ can be written as $f(\theta+\xi) / f(\theta)$

$$
\begin{array}{lc}
\text { iff } C \hat{f}(m)=e^{2 \pi \imath\langle m, \xi\rangle} \hat{f}(m) & \forall m \\
\text { iff } C=2^{2 \pi i\langle m, \xi\rangle} & \text { for some } m \in \mathbf{Z}^{v} .
\end{array}
$$

Thus we may solve (3.6) for smooth (analytic) $f$ for a given $g$ iff we can do it for $\mathrm{Cg}$ where

$$
C=e^{2 \pi i\langle m, \xi\rangle}, \quad \text { some } m \in \mathbf{Z}^{v} .
$$

We may summarize all of this as follows. 
3.8. If $\log g(\theta)$ is analytic then (3.6) may be solved for analytic $f(\theta)$, if

$$
\frac{1}{(2 \pi)^{v}} \int_{\pi^{v}} \log g(\theta) d \theta=2 \pi i(m+\langle n, \xi\rangle)
$$

for some $m \in \mathbf{Z}, n \in \mathbf{Z}^{v}$.

Notice that the points $m+\langle n, \xi\rangle$ with $m \in \mathbf{Z}, n \in \mathbf{Z}^{v}$ are dense in $\mathbf{R}$.

3.9. We will need to know that if the integral condition of (3.8) fails then one cannot solve (3.6), even in $L^{1}$.

Lemma. Suppose that the $g$ of (3.6) satisfies $\log |g| \in L^{1}$ and that $\int \log |g| \neq 0$; then (3.6) has no nonzero $L^{1}$ solutions.

Proof. Let $f \in L^{1}, f(\theta+\xi)=g(\theta) f(\theta)$. Iterating this gives

$$
g(\theta) g(\theta+\xi) \ldots g(\theta+(n-1) \xi) f(\theta)=f(\theta+n \xi) .
$$

Choose $n_{k} \rightarrow \infty$, so that $\left\|n_{k} \xi\right\| \rightarrow 0$; then

$$
f\left(\theta+n_{k} \xi\right) \rightarrow f(\theta) \text { a.e. . }
$$

Now $|\{\theta:|f(\theta)| \neq 0\}|>0$, so we see that

$$
g(\theta) g(\theta+\xi) \ldots g\left(\theta+\left(n_{k}-1\right) \xi\right) \rightarrow 1
$$

for $\theta$ in a set of positive measure. However

$$
\frac{1}{n} \sum_{r=0}^{n-1} \log |g(\theta+r \xi)| \rightarrow C \neq 0 \quad \text { for a.e. } \theta
$$

by the Birkhoff ergodic theorem. This clearly is a contradiction.

The above analysis may be applied to the problem of getting the point spectrum for the operator $H_{\varepsilon}$ of (3.1).

$$
p(\theta) f(\theta)+\varepsilon f(\theta+\xi)=\lambda f(\theta), \quad f \in L^{2}
$$

or

where

$$
\left.\begin{array}{rl}
f(\theta+\xi) & =g(\theta) f(\theta), \\
g(\theta) & =\frac{1}{\varepsilon}(\lambda-p(\theta)) .
\end{array}\right\}
$$

Define

$$
G(z)=\frac{1}{(2 \pi)^{v}} \int_{\pi^{v}} \log |z-p(\theta)| d \theta .
$$

Lemma 3.11. $G$ is subharmonic, continuous and harmonic outside of $[0,4 v]$. $G(z) \rightarrow \infty$ as $|z| \rightarrow \infty$ and $\beta=G(2 v) \leqq G(z)$ for all $z$.

Proposition 3.12. For $\varepsilon<\exp \{G(2 v)\}$, (3.1) has no point spectrum, while for $\varepsilon>\exp \{G(2 v)\},(3.1)$ has infinitely many localized eigenfunctions with eigenvalues dense on the lemniscate

$$
G(\lambda)=\log |\varepsilon|
$$


Proof.

$$
\frac{1}{(2 \pi)^{v}} \int_{\pi^{v}} \log |g(\theta)| d \theta=-\log |\varepsilon|+G(\lambda),
$$

thus if $\varepsilon<\exp \{\beta\}$, then

$$
\int \log |g|>0 \text {, }
$$

so by (3.9) there are no $L^{1}$ solutions, let alone $L^{2}$ solutions.

If $\varepsilon>\exp \{\beta\}$, then the lemniscate $P_{\varepsilon}=\{\lambda: G(\lambda)=\log |\varepsilon|\}$ is nonempty and looks something like Fig. 3.

Now for $\operatorname{Im}(\lambda) \neq 0, \lambda \in P_{\varepsilon}$, we see that $\log g(\theta)$ is analytic in $\theta$. Now if

$$
G_{1}(\lambda)=\frac{1}{(2 \pi)^{v}} \int_{\pi^{v}} \log \frac{1}{\varepsilon}(\lambda-p(\theta)) d \theta,
$$

then $G_{1}$ is analytic off the real axis and has constant real part along $P_{\varepsilon}$, so the imaginary part must vary and hence the set of $\lambda$ 's on $P_{\varepsilon}$ for which (3.8) holds is a countable dense subset of $P_{\varepsilon}$. To each such $\lambda(3.8)$ gives an analytic solution $f(\theta)$ to (3.10). So $\hat{f(m)}$ is a localized eigenstate.

Note that as $\varepsilon \rightarrow \infty$ the sets $P_{\varepsilon}$ approach circles of radius $\varepsilon$ about zero; this is not surprising in view of the perturbation of $\Delta$ off $\varepsilon V$.

Note. We have not been able to determine whether there is a point spectrum for the case of $\varepsilon=\exp \{\beta\}$.

We now analyze the rest of the spectrum, the inversion and construction of the extended states runs along lines similar to that of Sect.2; we run through the arguments briefly.

Consider

$$
f(\theta+\xi)-p_{\lambda}(\theta) f(\theta)=w(\theta), \quad w \in L^{2} .
$$

Denote $p_{\lambda}(\theta)$ by $g(\theta)$.

Lemma 3.14. If $c=\frac{1}{(2 \pi)^{v}} \int_{\pi^{v}} \log |g(\theta)| d \theta<0$ and $g(\theta) \neq 0$; then we can solve (3.13) for $f$ in terms of $w(\theta)$.

Proof. In this case the series of type (2.10) for $f(\theta)$ in terms of $w(\theta)$ is

$$
f(\theta)=w(\theta-\xi)+\sum_{k=1}^{\infty} g(\theta-\xi) \ldots g(\theta-k \xi) w(\theta-(k+1) \xi) .
$$

Now by Lemma 2.18,

so

$$
|g(\theta-\xi) \ldots g(\theta-k \xi)|^{1 / k} \rightarrow e^{c}<1 \text { uniformly, }
$$

$$
\|f\|_{2} \leqq\|w\|^{2}+\|w\|^{2} \sum_{k=1}^{\infty}\|g(\theta-\xi) \ldots g(\theta-k \xi)\|_{\infty} \leqq C_{5}\|w\|_{2}
$$

etc.

Notice that in the above we can allow $g$ to be zero at some points and still get the same result, since one can find $\psi(\theta)$ such that

$$
|g(\theta)| \leqq|\psi(\theta)| \text { and } \psi(\theta) \neq 0
$$

and $\int \log |\psi|<0$. We have 
Lemma 3.15. If $\int \log |g|<0$, then (3.13) has an $L^{2}$ solution for every $w \in L^{2}$.

Now suppose that $g(\theta) \neq 0$ and $\int \log |g|>0$, then to solve (3.13), we may divide by $g(\theta)$ to get the equation

$$
\frac{f(\theta+\xi)}{g(\theta)}-f(\theta)=\frac{w(\theta)}{g(\theta)} .
$$

The latter can be solved now by (3.14).

Interpreting the above lemmas we have shown that in the region

$$
G(\lambda)<\log |\varepsilon|
$$

we can invert $\lambda-H_{\varepsilon}$, i.e. $\lambda$ is in the resolvent of $H_{\varepsilon}$;

(ii) $G(\lambda)>\log |\varepsilon|$, and $g(\theta)$ nonvanishing (i.e. $\lambda \notin[0,4 v]$ ), then $\lambda$ is in the resolvent;

(iii) $G(\lambda)=\log |\varepsilon|$ has dense localized spectrum.

It remains to show that if

$$
\{G(\lambda)>\log |\varepsilon|\} \cap[0,4 v] \subset \sigma\left(H_{\varepsilon}\right),
$$

which we do by constructing a dense set of $\lambda$ 's for which we construct extended states. As before we need to control the product of the $p_{\lambda}$ 's. We will need some technical lemmas first.

Let $A \subset \Pi^{v}$ be closed with $\tilde{\mu}(A)=0$ (here $\tilde{\mu}$ denotes Lebesgue measure). For $\tau$ $>0$, let $A_{\tau}=\{x: d(x, A)<\tau\}$; then $\tilde{\mu}\left(A_{\tau}\right) \rightarrow 0$ as $\tau \rightarrow 0$. For our applications $A$ will be the zero set of a trigonometric polynomial and as such we will have an explicit rate

$$
\tilde{\mu}\left(A_{\varepsilon}\right)=0(\varepsilon) .
$$

Proposition 3.17. Let $A$ be as above; then if

$$
\begin{aligned}
& F=\left\{\xi \in \Pi^{v}: \exists C_{\xi}>0 \quad\right. \text { for which } \\
& \|n \xi-x\|>C_{\xi} n^{-\sigma} \quad \text { for some } \sigma>0 \\
& \text { and all } x \in A, n \geqq 1\} \text {, }
\end{aligned}
$$

then $\tilde{\mu}(F)=(2 \pi)^{v}$.

Proof. See appendix.

Now let $\lambda_{m}, m=\{1,2, \ldots\}$, be a dense sequence in $[0,4 v] \cap\{G(\lambda)>\log |\varepsilon|\}$. Let $A_{1}^{\prime}, A_{2}^{\prime}, \ldots$ be the corresponding zero sets $\left\{\theta: \lambda_{m}-p(\theta)=0\right\}=A_{m}^{\prime}$.

Let $\left\{b_{m}\right\}$ be a sequence of points, $b_{m} \in A_{m}^{\prime}$ and let

$$
A_{m}=b_{m}-A_{m}^{\prime} \text {. }
$$

For each $m$ we may construct $F_{m}$ as in (3.17) (with $A$ replaced by $A_{m}$ ). Let

$$
F_{1}=\bigcap_{m} F_{m} \quad \text { so that } \quad \tilde{\mu}\left(F_{1}\right)=(2 \pi)^{v} .
$$

Let $B$ be the set of strongly independent $\xi$. Now if $\xi \in F=F_{1} \cap B$, and also

$$
C=\int \log \left|\frac{\lambda_{m}-p(\theta)}{\varepsilon}\right| d \theta>0,
$$


we claim there is a measure $\mu$ (as usual) such that

$$
H_{\varepsilon} \mu=\lambda \mu \text {. }
$$

Proof. $g\left(b_{m}\right)=0$, so as in (2.4) set

then

$$
\mu=\sum_{j=0}^{\infty} \gamma_{j} \delta_{b_{m}+j \xi}(\theta)
$$

Take

$$
\gamma_{j-1}=-g\left(b_{m}+j \xi\right) \gamma_{j}
$$

$$
\begin{aligned}
& \gamma_{0}=1 ; \quad \gamma_{1}=-\frac{1}{g\left(b_{m}+\xi\right)} \cdots \\
& \gamma_{j}=\frac{(-1)^{j}}{g\left(b_{m}+\xi\right) \ldots g\left(b_{m}+j \xi\right)} .
\end{aligned}
$$

It remains to prove that

$$
\sum\left|\gamma_{j}\right|<\infty
$$

Now

$$
\left\|b_{m}+j \xi-x\right\| \geqq C_{\xi} j^{-\sigma}, \quad j \geqq 1, \sigma>0,
$$

and $x \in A_{m}^{\prime}=$ zero set of $g_{m}$. This follows by choice of $\xi \in F$. So the $\gamma_{j}$ 's are well defined. The above implies that

$$
\log \left|g\left(b_{m}+j \xi\right)\right| \geqq-C^{\prime \sigma} \log j \quad \text { for suitable } C^{\prime} .
$$

Let $h(\theta)=\log |g(\theta)|$, and let $\psi_{\varepsilon}$ be an approximate identity; let

$$
h_{\varepsilon}=\psi_{\varepsilon} * h \text {. }
$$

It is not difficult to show

$$
\sum_{r=1}^{n} h_{\varepsilon}(\theta+r \xi)=c n+B(n, \varepsilon)
$$

where $|B(n, \varepsilon)| \leqq \sum_{m \neq 0}\left|a_{m}(\varepsilon)\right||m|^{\sigma}$ (independent of $\theta$ ) (see (A.1)), and where the $a_{m}(\varepsilon)$ are the Fourier coefficients of $h_{\varepsilon}$. Thus

$$
\begin{aligned}
|B(n, \varepsilon)| & \leqq \sum\left|\hat{\psi}_{\varepsilon}(m)\right||\hat{h}(m)||m|^{\sigma} \\
& \leqq \sum^{\prime}\left|\hat{\psi}_{\varepsilon}(m)\right||m|^{\sigma} \\
& \sim \int_{|x| \geqq 1} \hat{\psi}(\varepsilon x) x^{\sigma} d x \\
& =\int_{1}^{\infty} \hat{\psi}(\varepsilon r) r^{\sigma+v-1} d r \\
& =C\left(\frac{1}{\varepsilon^{\sigma+v}}\right) .
\end{aligned}
$$


Next we estimate the difference

$$
\sum_{r=1}^{h}\left(h_{\varepsilon}(\theta+r \xi)-h(\theta+r \xi)\right) .
$$

To do so let $t$ be a parameter to be chosen and let

$$
C_{t}=\left(A_{m}\right)_{t}=\left\{x: d\left(x, A_{m}\right)<t\right\} .
$$

Outside of $C_{t}, \nabla h$ is bounded, indeed

so that

$$
|\nabla h| \leqq \frac{C_{6}}{t}, \quad \text { say }
$$

So

$$
\left|h_{\varepsilon}(\theta)-h(\theta)\right| \leqq \frac{\varepsilon C_{6}}{t} .
$$

$$
\begin{gathered}
\sum_{j=1}^{n} h\left(\theta_{0}+j \xi\right)=\sum_{j=1}^{n} h_{\varepsilon}\left(\theta_{0}+j \xi\right)+I+I I, \\
I=\sum_{\substack{j \xi \in C_{t}^{c} \\
1 \leqq j \leqq n}}\left(h-h_{\varepsilon}\right), \quad I I=\sum_{\substack{j \xi \in C_{t} \\
1 \leqq j \leqq n}}\left(h-h_{\varepsilon}\right) .
\end{gathered}
$$

Now $|I| \leqq C_{7} \frac{n \varepsilon}{t}$ by the above;

$$
\begin{gathered}
I I=\sum_{j \xi \in C_{t}} h\left(\theta_{0}+j \xi\right)-\sum_{j \xi \in C_{t}} h_{\varepsilon}\left(\theta_{0}+j \xi\right)=A+B \\
|A| \leqq 4 n t \max \left\{\left|h\left(\theta_{0}+j \xi\right)\right|\right\}
\end{gathered}
$$

(by uniform distribution ${ }^{1}$ and $\left.\lambda\left(C_{t}\right)=\mathrm{O}(t)\right) \leqq 4 n t C^{\prime} \sigma \log n \quad$ by (3.19).

Now $\quad\left\|h_{\varepsilon}\right\|_{\infty} \leqq\left\|\psi_{\varepsilon} * h\right\|_{\infty} \leqq\left\|\psi_{\varepsilon}\right\|_{r}\|h\|_{s}$,

where $1 / r+1 / s=1$ (we choose $r, s$ shortly). Also,

Therefore

$$
\left\|\psi_{\varepsilon}\right\|_{r} \sim\left(\frac{1}{\varepsilon}\right)^{v-v / r}
$$

So combining

$$
|B| \leqq 4 n t\left(\frac{1}{\varepsilon}\right)^{v(1-1 / r)}\|h\|_{s} .
$$

$$
\begin{gathered}
\sum_{j=1}^{n} h\left(\theta_{0}+j \xi\right)=n c+R \\
R=O\left(\varepsilon^{-(\sigma+v)}+\frac{n \varepsilon}{t}+4 n t C^{\prime \sigma} \log n+4 n t\left(\frac{1}{\varepsilon}\right)^{v(1-1 / r)}\|h\|_{s}\right) .
\end{gathered}
$$

Choosing now $r=2 v /(2 v-1), s=2 v, t=1 /(\log n)^{2}, \varepsilon=1 /(\log n)^{3}$, shows that

$$
R=O\left(\frac{n}{\sqrt{\log n}}\right) \text {. }
$$

1 The sequence $j \xi$ is uniformly distributed in $\Pi^{v}$ 
It follows that

$$
\left|\gamma_{j}\right|=O\left(e^{-c / 2}\right)^{j}
$$

so the series (3.18) converges.

Putting together all of these propositions of Sect. 3 we obtain the results claimed in 0.1 . The number $a_{v}$ corresponds to the point at which the spectrum moves from being $[0,4 v]$, and $b_{v}$ to the point where $[0,4 v]$ lies interior to $P_{\varepsilon}$. These numbers are

$$
\begin{aligned}
& a_{v}=\exp \{G(2 v)\}=\exp \left\{\frac{1}{(2 \pi)^{v}} \int_{\Pi^{v}} \log \left|2 v-2\left(\sum_{j=1}^{v} \cos \theta_{j}\right)\right| d \theta\right\}, \\
& b_{v}=\exp \{G(0)\}=\exp \left\{\frac{1}{(2 \pi)^{v}} \int_{\Pi^{v}} \log \left|2\left(\sum_{j=1}^{v} \cos \theta_{j}\right)\right| d \theta\right\} .
\end{aligned}
$$

In dimension $v=1, a_{v}=b_{v}=1$ so there is an abrupt phase change, and the level curves $G(\lambda)=\log |\varepsilon|, \varepsilon>1$, are easily calculated to be confocal ellipses with center $(2,0)$ and arcs $\varepsilon+\varepsilon^{-1}, \varepsilon^{-1}-\varepsilon$ (use Jensen's formula).

$$
\begin{aligned}
& a_{2}=1, \\
& b_{2} \approx 3.2, \\
& a_{3} \approx 1.6, \\
& b_{3} \approx 4.5 .
\end{aligned}
$$

We remark that everything that we have said about $\Delta+V, V=\exp \{2 \pi i\langle m, \xi\rangle\}$ can be done for an arbitrary constant coefficient operator $H=P+V, V$ as above, where $P=P\left(D_{1}, \ldots, D_{v}\right)$ is a polynomial in $D_{1}, \ldots, D_{v}$.

\section{Appendix}

\section{A.1. Proof of (2.24)}

Suppose $\log g$ is analytic. We carry out the details in dimension 1.

$$
h(\theta)=\log |g(\theta)|=\sum_{m} a_{m} e^{2 \pi i m \theta},
$$

where $a_{m}$ are of rapid decrease

$$
\begin{aligned}
\sum_{r=0}^{n-1} h(\theta+\alpha r) & =\sum_{m} a_{m} \sum_{r=0}^{n-1} e^{2 \pi i m(\theta+r \alpha)} \\
& =a_{0} n+\sum_{m} \frac{a_{m}\left(e^{2 \pi i n\langle m, \alpha\rangle}-1\right)}{e^{2 \pi i\langle m, \alpha\rangle}-1} e^{2 \pi i m \theta} \\
& =a_{0} n+B,
\end{aligned}
$$


and where

$$
\begin{gathered}
|B| \leqq \sum_{m} \frac{2\left|a_{m}\right|}{m^{-\sigma}} \leqq \sum_{m} 2\left|a_{m}\right| m^{\sigma} . \\
a_{0}=\frac{1}{(2 \pi)^{v}} \int_{\Pi^{v}} \log |g(\theta)| d \theta,
\end{gathered}
$$

which proves the lemma.

A.2. Proof of (2.25)

by strong independence.

$$
\begin{aligned}
\left|j \alpha \pm \frac{p}{q}+m\right| & =\frac{1}{q}|\alpha q j+p+q m| \\
& \geqq \frac{1}{q}(q j)^{-\sigma}
\end{aligned}
$$

The rest of the proof is just as the argument following (3.19).

\section{A.3. Proof of (3.17)}

Let $\sigma$ be fixed, $\varepsilon>0$ and

$$
B_{\varepsilon, n}=\left\{\xi:\|n \xi-x\|<\varepsilon n^{-\sigma} \quad \text { for some } \quad x \in A\right\} .
$$

For such a $\xi$ there is $m \in \mathbf{Z}^{v}$ such that

or

$$
|n \xi-x-m|<\varepsilon n^{-\sigma}
$$

$$
\left|\xi-\left(\frac{x+m}{n}\right)\right|<\varepsilon n^{-\sigma-1} .
$$

Since $\xi \in D=[0,2 \pi] \times \ldots \times[0,2 \pi]$ ( $v$ times) as is $x \in A \subset D$, it is clear that the $m$ 's involved satisfy

so if $\xi \in B_{\varepsilon, n}$ then

$$
|m| \leqq 2|n|
$$

$$
\xi \in \bigcup_{\substack{x, m \\|m| \leqq 2 n}} B_{\varepsilon n^{-\sigma-1}}\left(\frac{x+m}{n}\right) \subset \bigcup_{|m| \leqq 2 n}\left(\frac{A+m}{n}\right)_{\varepsilon n^{-\sigma-1}} .
$$

For each $m$

Thus

$$
\lambda\left(\frac{A+m}{n}\right)_{\varepsilon n^{-\sigma-1}} \leqq \varepsilon n^{-\sigma-1} .
$$

Thus

$$
\lambda\left(B_{\varepsilon, n}\right) \leqq \varepsilon n^{-\sigma-1+v}
$$

$$
\begin{aligned}
\lambda\left(\bigcup_{n} B_{\varepsilon, n}\right) & \leqq \varepsilon C \quad \text { if } \quad \sigma>v-1 \\
& \Rightarrow \lambda\left(\bigcap_{\varepsilon} \bigcup_{n} B_{\varepsilon, n}\right)=0 .
\end{aligned}
$$


Acknowledgement. I would like to thank P. Deift, Y. Katznelson, S. Klainerman and T. Spencer for many interesting and useful conversations and B. Simon for his encouragement and insights.

\section{References}

1. Aubry, S., Anré, G.: In: Colloquium on group theoretical methods in physics, Kiryat Anavim, Israel, 1979

2. Avron, J., Simon, B.: Almost periodic Hill's Equation and the rings of Saturn. Preprint (Cal. Tech.), 1981

3. Avron, J., Simon, B.: Cantor spectrum and limit periodic potentials. Preprint (Cal. Tech.), 1981

4. Goldshtein, Ya., Molchanov, S.A., Pastur, L.A.: Funct. anal. 11, 1 (1977)

5. Herstein, I.N.: Topics in Algebra, New York: Blaisdell, 1964.

6. Moser, J.: An Example of a Schrödinger Equation with almost periodic potential and Nowhere Dense Spectrum, E.T.H., Zürich, preprint, 1980

7. Moser, J.: Stable and random motions in dynamical system. Princeton Lecture Series 11, Princeton, N.S.: Princeton Univ. Press, 1973

8. Reed, M., Simon, B.: Methods of mathematical physics, Vol. 4., New York: Academic Press 1978

9. Sinai, Ya., Dinaburg, E.: Funct. Anal. Appl. 9, 279 (1976)

10. Thouless, D.J.: Phys. Rep. (Section C of Phys. Lett.) 13, No. 3, 93-142 (1974)

11. Katznelson, Y.: Israel J. Math. 33, 1 1-4 (1979)

12. Meyer, Y.: Algebraic numbers and harmonic analysis, p. 16. Amsterdam: North-Holland 1972

13. Gordon, A.Ya.: SUP. Math. Nauk 31, 257 (1976)

Communicated by B. Simon

Received June 5, 1981; in revised form December 7, 1981 
